\title{
Schultheiß Carl Förstner (1848-1939) aus Übrigshausen - Landtagsabgeordneter zwischen Deutscher Partei und Bauernbund
}

\author{
von Hans Peter Müller
}

Bis in die 1890er Jahre wurde die Parteienlandschaft des Königreichs Württemberg durch die klassischen, in den 1860er Jahren entstandenen Antipoden der Landespolitik, die demokratische Volkspartei und die nationalliberale Deutsche Partei (DP), geprägt. Die Demokraten sahen sich als Gralshüter der Revolutionsforderungen von 1848/49 und standen gegen eine „Verpreußung“ Württembergs. Nach der Reichsgründung von 1871 führte dieser Kurs zunächst ins politische Abseits. So dominierte die auf Bismarck fixierte, eher konservative als liberale DP im Verein mit der rein parlamentarischen Landespartei als quasi Regierungspartei das politische Leben, bis 1895 die Volkspartei als stärkste politische Kraft in die Zweite Kammer einzog. Inzwischen hatte sich auch eine Erweiterung des württembergischen Parteienspektrums vollzogen: Sowohl die Sozialdemokraten als auch die katholische Zentrumspartei waren in das Landesparlament eingezogen; mit der 1893 erfolgten Gründung des Bundes der Landwirte (BdL) auf Reichs- und Landesebene - hier wurde er zumeist als Bauernbund bezeichnet war eine weitere politische Kraft auf den Plan getreten. Während der BdL auf Reichsebene ihm genehme, meist konservative Kandidaten auf seine Ziele verpflichtete, etablierte sich seine württembergische Landesorganisation als Partei. Zwar unterstützte sie auch BdL-freundliche Bewerber aus der DP - eine für Förstner überaus günstige Konstellation -, nominierte jedoch gleichzeitig auch Kandidaten aus den eigenen Reihen. ${ }^{1}$

Der 1848 geborene Carl Rudolf Förstner amtierte seit 1875 in Nachfolge seines Vaters als - nicht fachlich vorgebildeter - Schultheiß seiner Heimatgemeinde Übrigshausen im Oberamt Hall. ${ }^{2}$ Er war Landwirt und gehörte über lange Jahre als Ausschussmitglied dem einflussreichen Landwirtschaftlichen Bezirksverein

1 Vgl. mit weiterer Literatur Hans-Peter Müller: Parteien und Politik in Hall 1860-1900. In: Hall im 19. Jahrhundert, eine württembergische Oberamtsstadt zwischen Vormärz und Jahrhundertwende (=Ausstellungskatalog), Sigmaringen 1991, S. 27-35. Zum Bauernbund ders.: Landwirtschaftliche Interessenvertretung und völkisch-antisemitische Ideologie. Der Bund der Landwirte/Bauernbund in Württemberg 1893-1918. In: ZWLG 53 (1994), S. 263-300.

2 Er amtierte bis 1929 und erhielt 1904 die Verdienstmedaille des Kronordens. Amtsblatt des Ministeriums des Innern 1904, S. 236. 
an. ${ }^{3}$ Gleichzeitig betrieb er eine Dampfziegelei, die um 1900 etwa 40 Beschäftigte zählte ${ }^{4}$ und für den ganz überwiegend landwirtschaftlich geprägten Bezirk ein herausragendes Unternehmen darstellte. Er gehörte so ganz eindeutig zum ,Establishment', das in aller Regel seine politische Heimat in der DP fand; Förstner gehörte der Partei seit den 1870er Jahren an. ${ }^{5}$ Dies hielt ihn jedoch nicht davon $\mathrm{ab}$, unmittelbar nach dessen Gründung auch dem Bauernbund als einer der ersten im Bezirk beizutreten und für den Bund zu werben. Er habe als Landwirt den Zusammenschluß der Bauern für dringend notwendig gehalten, um so bäuerliche Interessen besser vertreten zu sehen. ${ }^{6}$ Schon 1898 warb Förstner nachdrücklich für die Wahl des Bauernbunds-Bezirksvorsitzenden Frank aus Oberaspach in den Reichstag. ${ }^{7}$ Der dann nicht gewählte Frank war - wie eine Reihe weiterer Führer des württembergischen Bundes - zuvor Mitglied der DP gewesen. Die Allianz der sich beide betont national gebärdenden Gruppierungen DP und Bauernbund war so durchaus nachvollziehbar. Allerdings blieb die Doppelmitgliedschaft Förstners eine Besonderheit und war letztlich die Basis seiner politischen Laufbahn.

I. Vor dem Hintergrund dieser für ihn günstigen Ausgangslage entschloss er sich Ende 1900, als Kandidat zu den kommenden Landtagswahlen anzutreten. Er wurde von der DP nominiert, machte die Bewerbung jedoch von der Zusage des Bauernbundes abhängig, ihn als gemeinsamen Bewerber zu unterstützen. Erwartungsgemäß sprachen sich die Bündler für ihn aus. ${ }^{8}$ In einer ersten Wahlanzeige berief sich Förstner auf die in dringender Weise an ihn ergangene Aufforderung seiner Mitbürger in Stadt und Bezirk, denen er ein durchaus populäres Programm präsentierte. Als vornehmste Aufgabe sah er eine gerechte Steuerreform, die die höheren Einkommen stärker belaste, Mittelstand und Arbeiter dagegen entlaste; die großkapitalistischen Unternehmungen seien im Interesse der kleinen und mittleren Geschäfte stärker zu besteuern. Er wolle ferner für eine Revision der Verfassung eintreten, die Zweite Kammer müsse zur reinen Volkskammer werden. Schließlich plädierte er für eine Reform der Gemeindeordnung,

3 Vgl. dazu etwa Haller Tagblatt (fortan HT) Nr. 290 vom 12.12.1910.

4 Das Königreich Württemberg. Bd. 3, Jagstkreis. Stuttgart 1906, S. 255. Zur Ziegelei auch Frieder Krumrein, Eberhard Rau, Andreas Volk: Die Geschichte der früheren Gemeinde Übrigshausen. Ilshofen 2014, S. $341 \mathrm{ff}$.

5 In einer Rede betonte er 1902, er sei schon annähernd 30 Jahre Parteimitglied. HT Nr. 251 vom 27.10.1902. Mindestens zeitweise fungierte er dort als auswärtiges Ausschussmitglied der auf Bezirksebene organisierten Haller DP, ohne allerdings größere Aktivitäten zu entfalten. Vgl. dazu die (unvollständigen) Protokollbücher der Partei, StadtA Schwäbisch Hall 19/1088/1089.

6 HT Nr. 251 vom 27.10.1902.

7 HT Nr. 123 vom 29.5.1898 (Wählerversammlung des Bauernbundes). Dort wurde auch die erwähnte Doppelstrategie des Bundes - Aufstellung eigener, Unterstützung von DP-Kandidaten - artikuliert.

8 HT Nr. 263 vom 10.11.1900. Landesweit unterstützte der Bund etwa 20 Kandidaten der DP. HT Nr. 265 vom 13.11.1900 (Landesversammlung). 
d.h. vor allem für die Abschaffung lebenslänglicher Ortsvorsteher. Mit seinen weiteren Forderungen suchte er alle Bevölkerungskreise anzusprechen, wollte er doch Schutz und Förderung der einheimischen landwirtschaftlichen, gewerblichen und Handelsthätigkeit, eine Zentralgenossenschaftsbank solle günstige Kredite für Landwirtschaft und Kleingewerbe ermöglichen, Landwirtschaftskammern den Berufsstand besser repräsentieren. Gesetzliche Maßnahmen müssten schließlich die Auswüchse des Hausierwesens, der Konsumvereine und Warenhäuser eindämmen, Lohnarbeiter in Industrie und Landwirtschaft sollten gefördert, das Verkehrswesen besonders auf dem Land verbessert werden. ${ }^{9}$

Seinen zahlreichen Wahlversammlungen attestierte das deutschparteilich orientierte Bezirksblatt nicht nur ein sachliches Auftreten, sondern betonte auch, dass ihm ganz offenbar das Wohl des ganzen Volkes am Herzen liege. ${ }^{10}$ Ausführlich wurde über sein Programm berichtet und dabei auch präzisiert, dass er sich für das Projekt einer Kochertalbahn von Hall über Braunsbach nach Künzelsau einsetzen wolle. ${ }^{11}$ Während die Volkspartei das Zusammengehen der DP mit dem Bauernbund kritisierte, stieß diese Allianz, wie ein weiterer Versammlungsbericht betonte, insbesondere bei den ländlichen Wählern auf eine besondere Freude und lasse so das Beste erhoffen. ${ }^{12}$

Ungeachtet seiner günstigen Aussichten versäumte es das für Förstner agierende Wahlkomitee nicht, in einem langen Aufruf harsch mit den politischen Gegnern abzurechnen. Gegenüber dem SPD-Kandidaten Karl Krüger wurde der geradezu klassische Vorbehalt des bürgerlichen Establishments artikuliert: Seine Partei erstrebe offen den Umsturz der bestehenden Staats- und Gesellschaftsordnung. Dem volksparteilichen Kandidaten Professor Hoffmann warf man vor, seine Partei habe nach schönen Versprechungen die Steuerreform vereitelt. Demgegenüber sei Förstner ein Mann des besonnenen Fortschritts und von nationaler und bewährter liberaler Gesinnung, der als Bauernbundsmitglied auch die Besserstellung der hart bedrängten Landwirtschaft erstrebe. Mit seinen Erfahrungen als Schultheiß, Landwirt und Industrieller sei er der ideale Repräsentant aller Berufsklassen. ${ }^{13}$

Förstners absehbarer Wahlsieg am 5. Dezember fiel so überaus deutlich aus: Während 2175 Wähler für ihn votierten, erhielt sein volksparteilicher Gegner 1150, der Sozialdemokrat 724 Stimmen. ${ }^{14}$ Wie er 1902 auf einer DP-Versammlung erklärte, war er sich bewusst, dass das Hauptkontingent seiner Wähler aus 
den Reihen des Bauernbundes bzw. aus der ländlichen Wählerschaft kam. ${ }^{15} \mathrm{Er}$ hatte daher geplant, der DP-Fraktion im Landtag nur als Gast anzugehören, die DP bestand jedoch auf einer regulären Mitgliedschaft. ${ }^{16}$ Ein Teil der bauernbündlerischen Abgeordneten nahm dies zunächst mit Misstrauen und falschen Vermutungen auf. Er erklärte jedoch, deswegen nicht weniger bauernfreundlich zu sein, schließlich habe er als Landwirt und Unternehmer mit starkem Absatz an die ländliche Bevölkerung das größte Interesse am Wohlergehen der Landwirtschaft. Zudem herrsche bei der DP in Wirtschaftsfragen kein Parteizwang. Bezüglich des vom BdL geforderten höheren Zollschutzes für die Getreideeinfuhr - ohnehin ein Thema des Reichstages - gelte, dass er diesen zwar wünsche, aber weitergehende Forderungen als aussichtslos ansehe. In seiner langen Rede referierte er ausführlich über die Arbeit der Kammer und wurde vom Lokalblatt als äußerst sachkundig gelobt. ${ }^{17}$ Insgesamt war hier deutlich geworden, dass Förstner letztlich Abgeordneter von Gnaden des Bauernbunds war, eine Tatsache, die seine politische Zukunft entscheidend beeinflussen sollte.

Sein Wirken im Landtag war das eines Hinterbänklers; die ,große Politik ‘ war nicht sein Gebiet. Seine Themen waren solche mit lokalem bzw. regionalem oder landwirtschaftlichem Bezug. So plädierte er etwa am 28. Mai 1902 für einen verbesserten Modus bei der Gewährung von Ernteurlaub für Soldaten. ${ }^{18}$ Im Rahmen der schon vor der Jahrhundertwende landesweit einsetzenden Agitation zum Bau von Nebenbahnen schien ihm das Projekt einer von Hall ausgehenden Kocherbahn über Künzelsau hinaus ${ }^{19}$ die Möglichkeit zur Profilierung zu bieten. Nachdem jedoch 1892 eine Nebenbahn von Waldenburg nach Künzelsau errichtet wurde, verlor dieser Plan an Attraktivität. Zehn Jahre später brachte er den alten Plan im Landtag erneut zur Sprache: Er regte zunächst eine Weiterführung des Projekts Jagstfeld-Neuenstadt bis Künzelsau an und stellte schließlich in Aussicht, dass die Gemeinden von Hall abwärts bis Künzelsau wohl demnächst eine Bitte zum Bahnbau Hall-Braunsbach-Künzelsau an die Kammer richten würden. ${ }^{20}$

Erstaunlicherweise ruhten die Aktivitäten in dieser Sache dann - soweit feststellbar-bis 1906. Im Februar debattierte der Haller Gemeinderat das Projekt Kochertalbahn mit dem Tenor, dafür die Agitation wieder aufzunehmen und das entsprechende Komitee zu aktivieren. Allerdings stehe fest, dass die Stadt wegen des zu

15 HT Nr. 251 vom 27.10.1902.

16 DP-Protokolle, StadtA SHA 19/1089.

17 HT Nr. 251 vom 27.10.1902.

18 Landtagsprotokolle, S. 2265.

19 Vgl. dazu die Denkschrift „zur Erlangung einer Eisenbahn Hall-Künzelsau-Möckmühl“ vom Aug. 1888.

20 Landtagsprotokolle vom 30.1.1902, S. 1929, 23.5.1902, S. 2202. - Das Stadtarchiv Schwäb. Hall verwahrt unter der Signatur 21/1290 ein Gutachten über diesen Bau vom März 1903 (freundlicher Hinweis von Herrn Kratochvil, Enslingen), in dem jedoch weitere Hinweise auf dessen Weiterbehandlung fehlen. Lediglich die Aussage im Gemeinderat vom Februar 1906, man könne sich aus Kostengründen auf den Vorschlag nicht einlassen, scheint sich auf das Gutachten zu beziehen. HT Nr. 40 vom 17.2.1906. 
großen Aufwands kaum mit dessen Realisierung rechnen könne - es sei denn, der Staat oder eine Privatgesellschaft zeigten Interesse. Eine neue Komitee-Versammlung wurde angekündigt; Förstner sollte als Vorsitzender fungieren. ${ }^{21}$

Am 11. April 1906 tagte diese Versammlung, zu der zahlreiche Kochergemeinden zwischen Hall und Künzelsau Vertreter entsandt hatten. Förstner wollte den ihm angetragenen Komitee-Vorsitz zunächst nicht annehmen, erst nach seiner einstimmigen Wahl durch sämtliche Anwesenden stimmte er schließlich zu. Man war sich einig, dass die anzustrebende Verbindung Teil einer Durchgangsbahn von Hall über Künzelsau bis Jagstfeld werden sollte; hier war die Strecke Jagstfeld-Neuenstadt bereits projektiert. ${ }^{22}$ So gelte es, mit dem Komitee für den Bahnbau Künzelsau-Neuenstadt zu kooperieren, das bereits eine Denkschrift an die Regierung eingereicht hatte. Im Verein mit dem Haller Stadtschultheißen Hauber und einem Vertreter Künzelsaus sollte Förstner ebenfalls eine Denkschrift ausarbeiten. ${ }^{23}$ Die im Grunde unrealistischen und so nur halbherzigen Bemühungen sollten jedoch scheitern; das stets so stiefmütterlich behandelte Projekt der Kocherbahn war nach einem Zeitungsbericht von 1908 abermals durchgefallen. ${ }^{24}$

Inzwischen war der Bauernbund in Württemberg zu einer politischen Macht geworden. Längst gut organisiert und publizistisch präsent transportierte er seine agrarischen Forderungen und seine - später noch zu behandelnde - Ideologie über die Mitglieder hinaus ins Land; Ende 1906 hatten deren Zahl bereits über 22000 erreicht. ${ }^{25}$ Vor diesem Hintergrund zeichnete sich die Tendenz ab, möglichst viele eigene Kandidaten bzw. solche der eng verbündeten Konservativen ins Parlament zu entsenden. Schon 1903 hatte sich das Verhältnis zur DP abgekühlt: sie und die Volkspartei präsentierten zur Reichstagswahl 1903 im 11. und 13. württembergischen Wahlkreis (Hall und Crailsheim) gemeinsame Kandidaten, die jedoch gegen die erstarkten Bündler unterlagen. Die beiden liberalen Parteien begründeten ihren Schritt mit den Stadt und Land entzweienden „Gelüsten des

21 HT Nr. 40 vom 17.2.1906.

22 Vgl. Regierungsblatt für das Königreich Württemberg, Nr. 31 vom 22.11.1905, S. 275 f.

23 HT Nr. 86/1 und 87 vom 12. und 14.4.1906. Diese Denkschrift war jedoch nicht zu ermitteln; wahrscheinlich wurde sie nie erstellt. (Auch eine Anfrage beim StA Ludwigsburg zu den Hintergründen des Projekts lieferte kein Ergebnis: E-Mail v. 9.10.15, Az. 5-7512Sn). Immerhin bemühte sich Förstner, von den betroffenen Gemeinden Zusagen über die Übernahme der Grunderwerbungskosten zu erhalten. Vgl. dazu Archiv (Braunsbach-)Geislingen GA 248.

24 HT Nr. 271 vom 18.11.1908. Eine Verhandlung des Haller Bezirksrats vom März 1909 erläuterte als Grund die unverhältnismäßigen Kosten. Zur Schaffung besserer Verkehrsverhältnisse wurde nun eine Automobilverbindung von Hall nach Braunsbach angestrebt und auch realisiert. KrA SHA $1 \mathrm{~B} 26$.

25 Vgl. dazu Müller, Interessenvertretung (wie Anm. 1), S. 274 f. 
ostelbischen Junkertums" ${ }^{26}$ Auch wenn sich diese Bewertung auf die Reichspolitik bezog, dürfte die Entwicklung auf Förstner alarmierend gewirkt haben.

II. Tatsächlich kam zu den Landtagswahlen 1906 ein landesweites Bündnis zwischen DP und Bauernbund nicht mehr zustande. Vielmehr traten in 27 Wahlkreisen eigene Bauernbunds-Kandidaten - darunter vier Konservative - an. Nur in einigen Bezirken waren Absprachen auf Gegenseitigkeit mit der DP erfolgt. Dies war dann auch in Hall der Fall, wo sich der Bauernbund, offenbar mit Förstner zufrieden, für dessen Wiederwahl aussprach, ${ }^{27}$ nachdem die Haller DP schon im Januar 1906 für ein Festhalten an ihm plädiert hatte. ${ }^{28}$

Im Oktober nahm er die Kandidatur offiziell an und das Bezirksblatt berichtete, auf verschiedenen Versammlungen sei deutlich zum Ausdruck gekommen, dass die ihm zuvor entgegengebrachten Sympathien fortbestünden; er habe sein Mandat pflichtgetreu wahrgenommen. ${ }^{29}$ Förstner sprach in seiner Wahlanzeige zunächst von der Ueberwindung mancher Bedenken, um dann die erreichten grossen Reformwerke des letzten Landtags zu betonen. Auch dem neuen Parlament werde es nicht an wichtigen Vorlagen fehlen. Er nannte u. a. die Ergänzung der Steuerreform durch eine Vermögenssteuer und die staatliche Übernahme der Schullasten.

Ohne Voreingenommenheit und im Sinne eines besonnenen Fortschritts werde er für die Förderung aller Wirtschaftszweige eintreten und betonte dabei, dass sein Standpunkt den Wählern bekannt sei. ${ }^{30}$ Dennoch bereiste er auf seinen anschließenden Versammlungen den Bezirk intensiv. ${ }^{31}$ Das für ihn tätige Wahlkomitee beklagte etwa den im Parteienkampf aufgetretenen unheilvollen Gegensatz zwischen Stadt und Land um dann zu betonen, dass der vom Bauernbund unterstützte Förstner diese Kluft überbrücken werde. Zudem erheische sein bisheriges Wirken die Wiederwahl als eine Pflicht der Dankbarkeit. ${ }^{32}$

Schließlich betonte ein Aufruf der DP die Gegensätze zu den politischen Gegnern. Die verhüllten oder unverhüllten ,Endziele' der Sozialdemokratie trennten die DP für immer von dieser. Die Kandidatur eines bezirksfremden Bewerbers durch die verärgerte Volkspartei wurde als reine Demonstration abgetan. ${ }^{33}$ Durch die Unterstützung des Bauernbundes war am Sieg Förstners wie schon 1900 ernstlich wohl nicht zu zweifeln. Mit 2203 Stimmen votierten mehr als

26 Zit. nach ebd., S. 293.

27 HT Nr. 282 vom 1.12.1906.

28 HT Nr. 16/1 vom 20.1.1906.

29 HT Nr. 256 vom 1.11.1906.

30 HT Nr. 271 vom 19.11.1906.

31 Allerdings waren in der Presse nur Werbeanzeigen, jedoch keine Versammlungsberichte zu finden.

32 HT Nr. 281 vom 30.11.1906.

33 HT Nr. 284 vom 4.12.1906. 
doppelt so viele Wähler wie für den Sozialdemokraten Krüger für ihn; der volksparteiliche Kandidat konnte nur 469 Stimmen erreichen. ${ }^{34}$ Während das Haller Wahlergebnis das Fortbestehen der bisherigen Verhältnisse suggerierte, zeigte das Landesergebnis nach der Verfassungsänderung ${ }^{35}$ einen deutlichen Wandel: Nach dem Zentrum, der Volkspartei und der SPD rangierten Bauernbund und Konservative bereits vor der DP, der Umbruch der politischen Landschaft war evident geworden.

Förstner blieb in der Folgezeit bemüht, das für ihn essentielle gute Verhältnis zum Bauernbund zu erhalten. Während einer Versammlung des Bundes in Hall erläuterte er die Haltung der DP in Sachen einer Wertzuwachssteuer und lobte ausdrücklich den Einsatz seines Abgeordneten-Kollegen Schrempf für den Bauernstand. ${ }^{36}$

Seine parlamentarischen Aktivitäten blieben wie schon zuvor auf wenige Auftritte - zumeist im Interesse der Landwirtschaft - begrenzt. So plädierte er etwa für die Ausbildung von Laien als Geburtshelfer bei Haustieren ${ }^{37}$ und äußerte sich skeptisch zur Einführung der Schlachtviehversicherung; er wollte vielmehr die Ortsviehversicherungsvereine gestärkt sehen. ${ }^{38}$ Ferner monierte er, dass der neue Haller Oberamts-Tierarzt nunmehr auch die Bezirke Gaildorf und Backnang betreuen sollte. Er prognostizierte Unzufriedenheit der Viehbesitzer und eine Kostensteigerung durch höhere Reisekosten. ${ }^{39}$ Als Anwalt seines Bezirks wandte er sich schließlich in einer engagierten Rede gegen die drohende Aufhebung des Haller Landgerichts, für dessen Erhaltung bisher namhafte Opfer gebracht worden seien. Ein Verlust würde das historisch so bedeutsame Hall mit einem Schlag auf den Status einer gewöhnlichen Provinzstadt reduzieren. ${ }^{40}$

Inzwischen begannen sich die Beziehungen der Nationalliberalen zum BdL auf Dauer zu verschlechtern, wolle doch dessen üble Führung die Bauern für ihre großbäuerlichen Interessen instrumentalisieren. ${ }^{41}$ Gleichzeitig wurde auf einer Stuttgarter Vertreterversammlung der DP die Annäherung der liberalen Partei-

34 HT Nr. 286 vom 6.12.1906.

35 Die gegen das Zentrum 1906 durchgesetzte Reform machte die Zweite Kammer zur reinen Volkskammer, in die nun zusätzlich zu den Abgeordneten der Bezirke und der „guten Städte“ 17 in den sog. Proporzwahlen ermittelte Vertreter einzogen. Vgl. zur Verfassungsreform etwa Andreas Gawatz: Wahlkämpfe in Württemberg: Landtags- und Reichstagswahlen beim Übergang zum politischen Massenmarkt 1889-1912 (Beiträge zur Geschichte des Parlamentarismus und der politischen Parteien 128, zugl. Diss. Tübingen 1999). Düsseldorf 2001, S. 40 ff., Frank Raberg: Biographisches Handbuch der württembergischen Landtagsabgeordneten 1815-1933. Stuttgart 2001, S. XXVIff. - Auch Förstner hatte während einer gemeinsamen Versammlung von DP und Volkspartei in Hall die Zentrumshaltung verurteilt. HT Nr. 152 vom 2.7.1904.

36 HT Nr. 6 vom 9.1.1908.

37 Landtagsprotokolle vom 9.7.1909, S. 5700.

38 Landtagsprotokolle vom 14.7.1909, S. 5792.

39 Landtagsprotokolle vom 5.4.1910, S. $6411 \mathrm{f}$.

40 Landtagsprotokolle vom 1.8.1911, S. 1727 f. Vgl. auch HT Nr.. 179 vom 3.8.1911.

41 Sommerfest der DP im 5. württembergischen Reichstagswahlkreis. HT Nr. 166 vom 20.7.1909. 
en festgestellt und begrü $\beta t,{ }^{42}$ während der Bauernbund Verdächtigungen seiner Reichsführung energisch zurückwies. ${ }^{43}$ Der Prozess von Abkehr und Annäherung im Parteienspektrum setzte sich fort. Württembergs DP-Parteiführer Hieber konstatierte ein Zusammenrücken von Bauernbund und Zentrum, während zwischen seiner Partei und der Volkspartei inzwischen ein freundlicheres Verhältnis bestehe; die Schärfe früherer Differenzen habe sich abgeschliffen. ${ }^{44}$ In einer weiteren DP-Versammlung wurde ein Block von Zentrum und Bauernbund gegen den Liberalismus konstatiert; dem Bauernbund eine Lösung von den ostelbischen Agrariern empfohlen. ${ }^{45}$ Darauf folgte der Vorwurf, die beiden liberalen Parteien hätten durch Lüge, Verleumdung und Verhetzung gegen den Bund agitiert. ${ }^{46}$ Schließlich lobte die Volkspartei die Frontstellung gegen rechts durch die DP, ${ }^{47}$ von der dann betont wurde, man erstrebe ein freundnachbarliches Verhältnis zur Volkspartei. ${ }^{48}$ Als Resultat dieser Entwicklung schlossen die beiden Parteien im November 1910 im Hinblick auf die Reichstagswahlen Anfang 1912 ein landesweites Wahlabkommen, in dem festgeschrieben wurde, dass man in keinem Wahlkreis gegeneinander antrete. ${ }^{49}$ Ein Pressekommentar meinte dazu, man sei wohl weniger einer inneren Herzenszuneigung als dem Zwange der Not gefolgt. ${ }^{50}$

Die hier geschilderte Entwicklung gibt Veranlassung, näher auf die Ideologie des BdL einzugehen. Er hatte auf Reichsebene „den Charakter einer bürokratisierten, undemokratisch und illiberal gesinnten plebejischen agrarischen Massenbewegung unter der Leitung von Landjunker-Demagogen und Berufsfunktionären" ". ${ }^{51}$ Auch wenn der Bund in Württemberg nicht von Junkern geführt wurde, vertraten seine Reichstagsabgeordneten schnörkellos den Kurs der Reichsführung, d.h. großagrarische Interessen. Während der Bund in Württemberg durchaus auch klein- und mittelbäuerliche Anliegen artikulierte und das Bauerntum und den Mittelstand glorifizierte, war sein ideologisches Weltbild identisch mit dem der Reichsführung. Dazu gehörte der Kampf gegen die Linken, gegen Sozialdemokratie und Volkspartei, die etwa als „Judenschutztruppe“ diffamiert wurden. In seinen Publikationsorganen wurde hemmungslos gegen die Juden polemisiert - sowohl als geldgierige Kapitalisten als auch als Führer

42 HT Nr. 279 vom 29.11.1909.

43 HT Nr. 304 vom 29.12.1909.

44 HT Nr. 305 vom 30.12.1909.

45 HT Nr. 96 vom 27.4.1910.

46 HT Nr. 111 vom 17.5.1910.

47 HT Nr. 170 vom 25.7.1910.

48 HT Nr. 183/1 vom 9.8.1910. Vgl. auch HT Nr. 237/2 vom 11.10.1910.

49 HT Nr. 271/1 vom 19.11.1910.

50 Zit. nach HT Nr. 273 vom 22.11.1910.

51 Hans Rosenberg: Zur sozialen Funktion der Agrarpolitik im Zweiten Reich. In: ders.: Probleme der deutschen Sozialgeschichte. Frankfurt/M. 1969, S. 66 f. 
der Sozialdemokratie. ${ }^{52}$ Letztlich hatte diese Propaganda den Antisemitismus in Württemberg hoffähig gemacht.

Die oben skizzierte liberale Annäherung wird nur vor dem Hintergrund des bündlerischen Wirkens verständlich, das letztlich mit liberalen Grundüberzeugungen nicht kompatibel war.

Zudem fungierten Volkspartei und DP als politische Heimat der jüdischen Deutschen und mussten beide den Einbruch des erstarkenden Bauernbundes in ihr ländliches Wählerpotenzial fürchten. Schließlich ist zu konstatieren, dass innerparteiliche Entwicklungen den liberalen Annäherungsprozess begünstigten: die $\mathrm{DP}^{53}$ war nicht zuletzt unter dem Einfluss der Jungliberalen nach links gerückt, während bei der Volkspartei inzwischen die alten demokratischen Grundsätze in den Hintergrund getreten waren. ${ }^{54}$ Dieser Trend wurde durch die linksliberale Einigung noch verstärkt. ${ }^{55}$ So konnte der Bauernbund 1912 feststellen, dass zwischen Deutscher Partei und Volkspartei kaum mehr ein Unterschied besteht. ${ }^{56}$

III. Sicherlich nahm Förstner die skizzierte Entwicklung, die er jedoch nicht beeinflussen konnte, mit Sorgen zur Kenntnis. Dennoch war Anfang September 1912 im Bezirksblatt zu lesen, die DP wolle auch für die kommenden Landtagswahlen unbedingt an ihm festhalten, habe er doch das volle Vertrauen des Bezirks errungen, das sich auch auf die Volkspartei erstrecke. Einer zu ihm entsandten Abordnung habe er, nach Ueberwindung verschiedener Bedenken, die Bereitschaft zur erneuten Kandidatur erklärt. Euphorisch wurde erklärt, Förstner ist unser Mann. ${ }^{57}$

52 Vgl. zum Obigen Müller: Interessenvertretung (wie Anm. 1), S. 282-287 u. passim, James C. Hunt: The „Egalitarianism of the Right“: The Agrarian League in Southwest Germany 1893-1914. In: Journal of Contemporary History 10 (1975), S. 513-530, zum Antisemitismus S. 521, Stefan Biland: Die (Deutsch-)Konservative Partei und der Bund der Landwirte in Württemberg vor 1914 (Stuttgarter historische Studien zur Landes- und Wirtschaftsgeschichte 2). Stuttgart 2002, Reinhold Weber: Bürgerpartei und Bauernbund in Württemberg (1895-1933) (Beiträge zur Geschichte des Parlamentarismus und der politischen Parteien 141). Düsseldorf 2004.

53 Sie firmierte inzwischen als Nationalliberale Partei - Deutsche Partei in Württemberg.

54 Vgl. dazu etwa der Artikel von L .Elm: Süd-Deutsche Volkspartei (SDVp) 1868-1910. In: Lexikon zur Parteiengeschichte, Bd. 4. Leipzig 1986, hier S. 175.

551910 fusionierten die Freisinnige Vereinigung und die Freisinnige Volkspartei mit der württembergischen bzw. süddeutschen Volkspartei zur Fortschrittlichen Volkspartei. - Vgl. zur Annäherung von Volkspartei und Nationalliberalen etwa James C. Hunt: The People's Party in Württemberg and Southern Germany 1890-1914 (Stuttgarter Beiträge zur Geschichte und Politik 9). Stuttgart 1975, S. $202 \mathrm{f}$ und passim.

56 Theodor Körner: Wahl-Handbuch. Zum Gebrauch und zur Orientierung bei den Landtagswahlen [...]. Stuttgart 1912, S. 11 (i. O. teilweise gesperrt).

57 HT Nr. 205 vom 2.9.1912. - Während das liberale Wahlabkommen sich auch auf die kommende 
Etwa vier Wochen später folgte die Antwort des Bauernbunds. Eine Vertreterversammlung des Bezirks Hall erklärte, nachdem er eine Kandidatur für die Liberalen angenommen habe und so gegen den Bund stehe, sei seine Zugehörigkeit zu diesem verwirkt. Man werde nun das Weitere in die Wege leiten. ${ }^{58}$ Dieser Ankündigung folgte die Nominierung des langjährigen Schultheißen Carl Stiefel aus Wolpertshausen als Kandidat des Bauernbundes. Nach seinen bisherigen Wahlerfahrungen musste nun Förstner den Verlust seines Mandates befürchten.

Ende Oktober zog er seine Kandidatur zurück, allerdings wurde mitgeteilt, die seinerzeit geäußerten Bedenken seien medizinischer Art gewesen. Seine $\mathrm{Ge}$ sundheitsverhältnisse hätten sich zwischenzeitlich so verschlechtert, dass er einen Stuttgarter Spezialarzt für Nervenkrankheiten konsultieren musste. Dieser habe ihm dringend geraten, sich den Anstrengungen und Aufregungen eines Wahlkampfes nicht zu unterziehen. So sei sein Verzicht schon aus familiären Gründen erfolgt. Die DP zeigte Verständnis und betonte, er dürfe sich allezeit des Dankes für seine 12-jährige Tätigkeit in treuester und gewissenhaftester Pflichterfüllung sicher sein. Während nun mit dem Jagstzeller Landwirt Schaible ein bezirksfremder liberaler Kandidat nominiert wurde, ${ }^{59}$ war politisch Informierten sicherlich klar, dass das Ende der politischen Karriere Förstners wohl nicht nur medizinische Gründe hatte. Auf einer Wahlversammlung in Übrigshausen erklärte Förstner solidarisch seine volle Uebereinstimmung mit Schaible und teilte zugleich mit, dass er aus dem Bauernbund ausgetreten sei. ${ }^{60}$

Die Wahl endete schließlich mit einem Triumph des Bauernbundes: Während dessen Kandidat Stiefel 2989 Stimmen erzielte, votierten für Schaible nur 912, für den Sozialdemokraten Krüger 973 Wähler. Das Bezirksblatt kommentierte, das Ergebnis sei nach der Reichstagswahl vom Beginn des Jahres kaum überraschend gewesen, zudem seien die Nationalliberalen unter den ungünstigsten Verhältnissen angetreten. ${ }^{61}$

Landtagswahl erstreckte und die DP so mit Widerstand aus dem Bauernbund rechnen musste, hoffte man dort dennoch offenbar auf gute Chancen des populären Förstner.

58 HT Nr. 232 vom 3.10.1912.

59 HT Nr. 251 vom 25.10.1912.

60 HT Nr. 265 vom 11.11.1912. Seine Austrittsgründe wurden in dem Zeitungsbericht nicht konkret formuliert. Allerdings warf Förstner einen Blick auf die Gründung des Bundes und dessen Fortentwicklung. Daraus ist zu schließen, dass er diese - oben skizzierte - Entwicklung nicht länger mittragen wollte. Er hatte bereits Anfang 1910 seinen Austritt aus dem Bauernbund in Aussicht gestellt. Protokolle der Haller DP, StadtA Schwäbisch Hall 19/1089.

61 HT Nr. 270 vom 16., Nr. 271 vom 18.11.1912 (Kommentar). 


\begin{abstract}
$* * *$
Wie bereits erwähnt, belegen Förstners Reden im Parlament, dass er sich lediglich als Anwalt seines Heimatbezirks bzw. landwirtschaftlicher Interessen sah. Weder , die große Politik' noch herausragende Aktivitäten im Parteileben waren Sache des beruflich stark Engagierten. Seine Mandatsgewinne 1900 und 1906 sind ganz und gar der Unterstützung durch den Bauernbund zuzuschreiben. War dieses Lavieren zwischen zwei Parteien zunächst unproblematisch, so schuf die veränderte politische Großwetterlage im Vorfeld der Wahlen von 1912 eine neue Situation und sorgte für ein abruptes Ende seiner parlamentarischen Laufbahn. Mit seiner sozusagen auch politischen Krankheit vermied er die absehbare Niederlage. $\mathrm{Zu}$ seinen Gunsten darf angenommen werden, dass sein früher Beitritt zum Bauernbund dem Ziel entsprang, im Sinne der Landwirtschaft zu wirken. Erst im Laufe der Zeit erkannte er die brunnenvergiftende Wirkung der bündlerischen Ideologie, mit der er sich, soweit zu sehen ist, nicht identifizierte. Vielmehr erscheinen seine wiederholten Beteuerungen, einem , besonnenen Fortschritt' verpflichtet zu sein durchaus glaubwürdig. Dass er schließlich - wenn auch spät - den Bauerbund verließ, spricht für ihn.
\end{abstract}

Das Wahlergebnis seines Nachfolge-Kandidaten zeigt schließlich überdeutlich, dass der Bauernbund inzwischen zur stärksten politischen Kraft weit über den Haller Bezirk hinaus geworden war. ${ }^{62}$ Dieser sich nach 1918 fortsetzende Trend sollte schließlich in der Endphase der Weimarer Republik verheerende Folgen zeigen; die bündlerische Ideologie hatte im heutigen Gebiet des Landkreises Schwäbisch Hall das Feld für den Nationalsozialismus vorbereitet. ${ }^{63}$

Der Prozess der politischen Umgruppierung beinhaltete den Niedergang des Liberalismus und den Aufstieg der neuen, erst in den 1890er Jahren in die politische Arena getretenen Parteien, die zugleich den „Durchbruch des modernen Parteiwesens “ dokumentierten. ${ }^{64}$ Im Verlauf dieses Prozesses war Förstner während zweier Wahlperioden sozusagen erfolgreich mitgeschwommen. Schon vor 1912 zeichnete sich dann ab, dass seine Strategie keine Perspektive mehr bot.

62 Nach dem Abschluss der Landtagswahlen standen sich im Landtag zwei gleichstarke Gruppierungen gegenüber. Stärkste Partei war das Zentrum mit 26 Sitzen, gefolgt vom Bauernbund, der mit seinem Dauerpartner, den Konservativen, über 20 Mandate verfügte. Als schwarz-blauer Block standen sie gegen die liberalen Wahlverlierer, d.h. die Volkspartei mit 19 und die Nationalliberalen mit 10 Sitzen sowie die Sozialdemokraten mit 17 Mandaten.

63 Vgl. dazu Hans-Peter Müller: Republik und Diktatur. In: Der Landkreis Schwäbisch Hall, Bd. 1. Ostfildern 2005, S. $77 \mathrm{ff}$.

64 Gawatz: Wahlkämpfe (wie Anm. 35), S. 116. 